\title{
The Impact of Transportation Disruptions on Performance of E-Collaboration Supply Chain
}

\author{
Tianjian Yang and Jun Wu \\ School of Economics and Management, Beijing University of Posts and \\ Telecommunications, Beijing 100876, P.R. China frankyti@,sohu.com
}

\begin{abstract}
In this paper, we analyze the impact of a transportation disruption on supply chain performance by using system dynamics simulation. Performances of three different supply chains with Internet e-collaboration tools are compared with the assumption that a transportation disruption occurs within two echelons in a five-echelon supply chain. Numerical results are shown to reveal that from Non-collaborative supply chain to Collaborative Forecasting and Collaborative Planning (or VMI) supply chains more and more robust is revealed under certain transportation disasters.
\end{abstract}

Keywords: Supply chain, Supply chain risk management, Disruption management, System dynamics, Transportation disruption

\section{INTRODUCTION}

The vulnerability of supply chains has undoubtedly received more attention since the attacks on the World Trade Centers on September 11, 2001, even though supply chains have always been faced with assessing their vulnerabilities and managing risk. Risks faced by supply chains are quite diverse, arising from sources both within and external to the supply chain. Christopher (2005) defined SCRM (supply chain risk management) as "the management of supply chain risks through coordination or collaboration among the supply chain partners so as to ensure profitability and continuity." Based on the definitions of SCRM, it appears that one can address the issue of SCRM along two dimensions: 1.Supply Chain Risk-operational risks or disruption Risks; 2.Mitigation Approach - supply management, demand management, product management, or information management.

We find that existing quantitative models are designed for managing operational risks primarily, not disruption risks. Since there are few supply chain management models for managing disruption risks, we would like to look into this field.

This paper investigates how a transportation disruption affects the supply chain performance of traditional supply chain and e-collaboration supply chains. Applying system dynamics simulation, this study determines how each of these structures responds to a transportation disruption at certain echelon in the supply chain. Supply chain response is measured by the number of service level, inventory fluctuations. Finally, this paper suggests strategies for mitigating the risk from a transportation disruption. 


\section{MODEL DESCRIPTION}

We use the system dynamics model which Oscar Rubiano Ovalle [5] developed.

\subsection{Non-collaborative Supply Chain}

When collaboration does not exist in the SC, an inventory manager only has operative information about the order placed by its direct downstream partner(s). And the causal diagram of the NC (Non-collaborative supply chain) system is shown in Figure 1 .

$$
\begin{gathered}
\hat{\mu}_{t}^{i}=\alpha^{i} D_{t-1}^{i+1}+\left(1-\alpha^{i}\right) \hat{\mu}_{t-1}^{i} \\
O P_{t}^{i}=\operatorname{Max}\left(\hat{\mu}_{t}^{i}+\beta_{s}\left(\hat{\mu}_{t}^{i} s s^{i}-Y_{t}^{i}\right)+\beta_{S L}\left(\hat{\mu}_{t}^{i} L^{i}-P_{t}^{i}\right)-B_{t-1}^{i-1}, 0\right)
\end{gathered}
$$

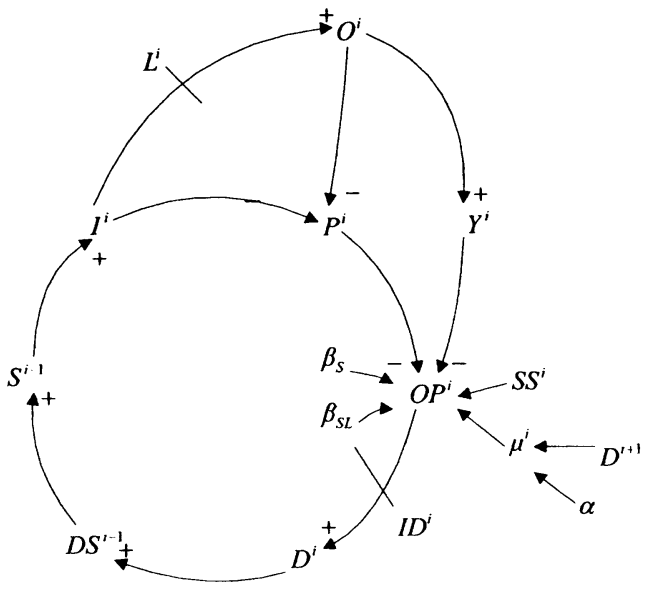

Figure 1. Causal Diagram of the NC System

\subsection{Collaborative Forecasting Supply Chain}

In this kind of collaboration, Eq. (1) is replaced by the following formulation:

$$
\begin{gathered}
\hat{\mu}_{t}^{i}=\hat{\mu}_{t}^{n}, \forall i=1, \ldots, n ; \\
\hat{\mu}_{t}^{i}=\alpha^{n} D_{t-1}^{\text {customer }}+\left(1-\alpha^{n}\right) \hat{\mu}_{t-1}^{n}, 0<\alpha^{n} \leq 1, \forall i
\end{gathered}
$$


The Impact of Transportation Disruptions on Performance of E-Collaboration Supply

Chain 665

and $D_{t-1}^{\text {customer }}$ is the last time period demand for the end customer of the chain.

Once the new Firm forecast is obtained, the orders are calculated as in (2).

Fig. 2 shows the unique modification to the causal loop and the stock and flow diagrams.

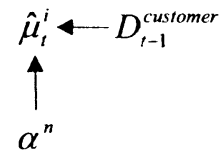

Figure 2. Forms Forecast in Collaborative Forecasting Process

\subsection{Collaborative Planning and VMI Supply Chain}

For the case, (3) and (4) are still applicable, but the following formulation (5) is introduced, replacing (2).

$$
\begin{gathered}
O P_{t}^{i}=\operatorname{Max}\left(\hat{\mu}_{t}^{i}+\beta_{s}\left(\hat{\mu}_{t}^{i} s s^{i}-Y_{t}^{i}\right)+\beta_{S L}\left(\hat{\mu}_{t}^{i} L^{i}-P_{t}^{i}\right)-B_{t-1}^{i-1}+i b_{t}^{i}, 0\right) \\
i b_{t}^{i}=\hat{\mu}_{t}^{i}\left(s s^{i+1}+L^{i+1}\right)-\left(Y_{t}^{i+1}+P_{t}^{i+1}\right)+i b_{t}^{i+1}
\end{gathered}
$$

These new relationships are included in the causal loop diagram (Figure 3). Note that no delay time exists between $O P^{i}$ and $D_{i}$ variables.

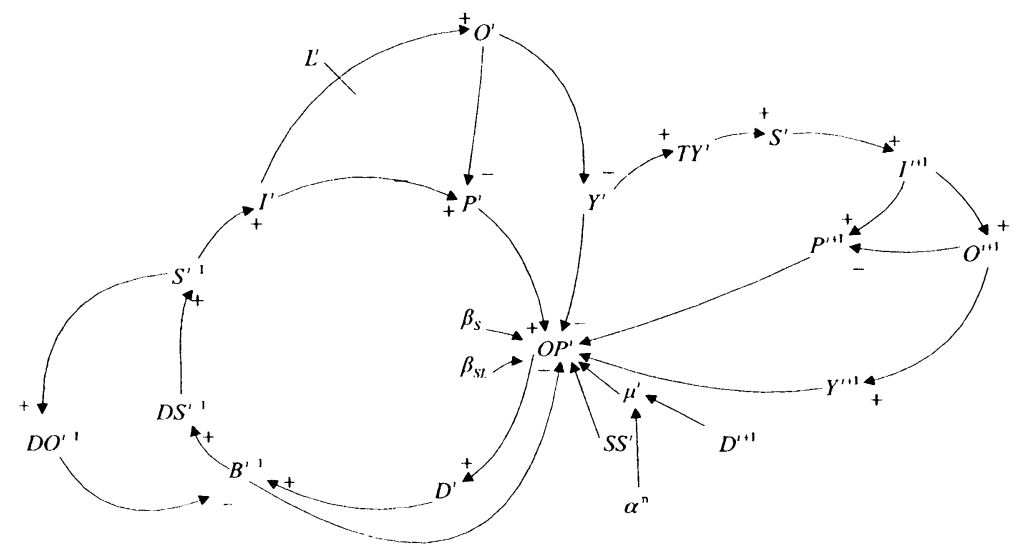

Figure 3. Causal Loop Diagram of the CP and VMI Structure 


\section{SIMULATION RESULTS}

3 types of models (NC, CF and CP) are built, and each of them is a 5-echelon supply chain. And 3 different input functions are tried on the system dynamics models, and it is assumed that transportation should be disrupted between the wholesaler and retailer when 100 to 104 weeks arrive. We focus on inventory and service level fluctuation in each case.

The experiment results are as follows: curve 1 donates the NC structure, curve 2 donates CF structure and No.3 donates the CP and VMI structure.

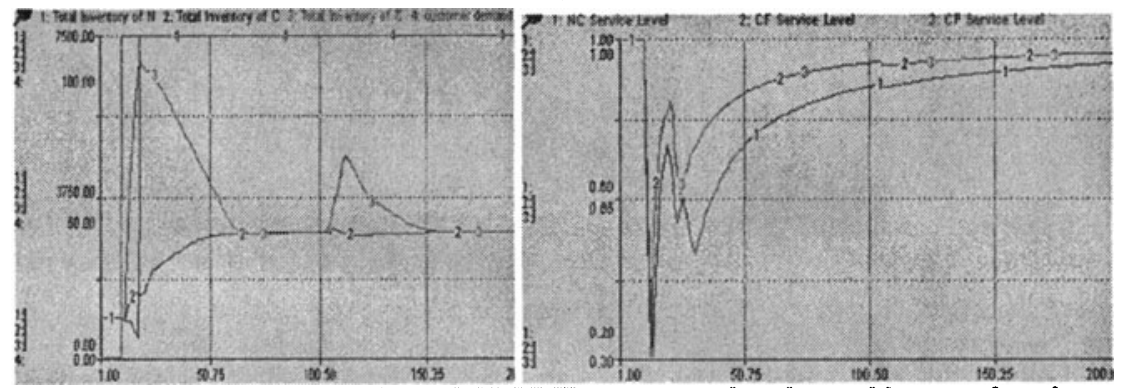

Figure 4. Comparison of NC, CF and CP(VMI) structure when demand is a step function
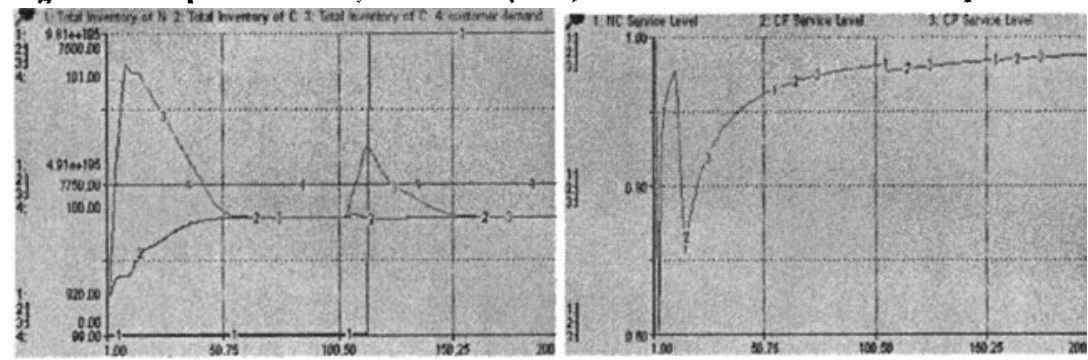

Figure 5. Comparison of $\mathrm{NC}, \mathrm{CF}$ and $\mathrm{CP}(\mathrm{VMI})$ structure when demand is constant
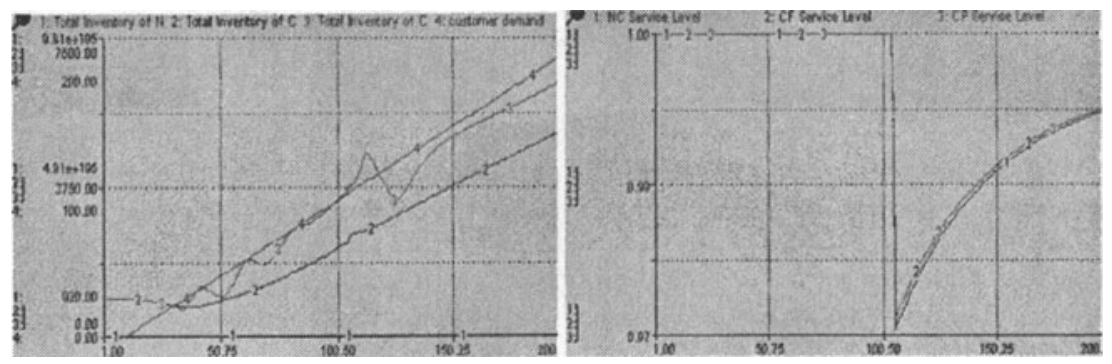

Figure 6. Comparison of NC, CF and CP (VMI) structure when demand is a ramp function 


\section{DISCUSSION AND CONCLUSIONS}

According to the experiment results, a few suggestions have been revealed in a calamitous supply chain:

The diagrams show that the NC structure have much more average inventory than the other two. And the CF structure has the least inventory. But we should note that the CP structure's inventory exceeds that of the CF only when the inventory fluctuation is not in a stable state.

The CP structure has the best service level. Although the NC has the most average inventory, the $\mathrm{CP}$ structure has the best service level. But we note that the CF structure is almost as good as the CP in most cases.

The CP structure is the most sensitive. It responds to the disruption by adding to inventory quickly and also responds to the transportation recovery by reducing the inventory quickly. By contrast, the CF structure respond by a much more gentle way, that is to say, the inventory rises and falls little by little. Different from the two, the $\mathrm{NC}$ inventory may keep fixed for a long while and suddenly go up a very, very high step.

Although a transportation disruption may lead to service level drops in all the three supply chain structure, we can say $\mathrm{CP}$ is the most robust of the three, with good emergency handling mechanics and the best service level.

\section{REFERENCES}

1. C.S. Tang, Perspective in supply chain risk management, Int. J. Production Economics. Volume 103, Number 2, pp.451-488, (2006).

2. A. Otto and $\mathbf{H}$. Kotzab, Does supply chain management really pay? Six perspectives to measure the performance of managing a supply chain, European Journal of Operational Research. Volume 144, Number 2, pp.306-320, (2003).

3. M.C. Wilson, The impact of transportation disruptions on supply chain performance, Transportation Research Part E: Logistics and Transportation Review. Volume 43, Number 4, pp.295-320, (2007).

4. D. Vlachos, P. Georgiadis, and E. Iakovou, A system dynamics model for dynamic capacity planning of remanufacturing in closed-loop supply chains, Computers \& Operations Research. Volume 34, Number 2, pp 367-394, (2007).

5. O.R. Ovalle and A.C. Marquez, The effectiveness of using e-collaboration tools in the supply chain: an assessment study with system dynamics, Journal of Purchasing \& Supply Management. Volume 9, Number 4, pp.151-163, (2003).

6. S.M. Disney, A.T. Potter, and B.M. Gardner, The impact of vendor managed inventory on transport operations, Transportation Research (Part E). Volume 39, Number 5, pp.363-380, (2003). 